\title{
LES FUTURES MISSIONS SPATIALES DÉDIÉES aux planètes extrasolaires
}

\author{
Pierre BAUDOZ \\ Laboratoire d'Études Spatiales \\ et d'Instrumentation en \\ Astrophysique (LESIA), \\ Observatoire de Paris, Université \\ PSL, CNRS, Sorbonne Université, \\ Univ. Paris Diderot, Sorbonne \\ Paris Cité, 5 place Jules Janssen, \\ 92195 Meudon, France \\ pierre.baudoz@obspm.fr
}

\section{Mille milliards de mille planètes !}

Depuis leur découverte au début des années 1990, des planètes extrasolaires ont été trouvées autour de tous les types d'étoiles, des plus communes (semblables au Soleil) aux étoiles plus exotiques comme les étoiles à neutrons en passant par des étoiles doubles ou multiples. Les planètes étant un résidu du processus de formation stellaire, elles semblent omniprésentes dans l'univers. Les estimations statistiques basées sur les 4000 planètes connues indiquent qu'en moyenne, chaque étoile de notre galaxie pourrait héberger au moins un compagnon planétaire [1]. Avec les quelques centaines de milliards d'étoiles dans notre galaxie, on est en dessous du décompte du capitaine Haddock mais si l'on élargit notre vision aux quelques 100 milliards de galaxies de notre univers, le capitaine risque d'être loin du compte. Pour l'instant, seules les planètes de notre galaxie ont été détectées mais, d'ici quelques années, il est probable que nous observions des exoplanètes dans les galaxies les plus proches, notamment avec les futurs télescopes géants de plus de $30 \mathrm{~m}$ de diamètre.

Les planètes observées jusqu'à aujourd'hui l'ont été principalement de manière indirecte, c'est-à-dire par l'observation de l'effet de la planète sur la lumière reçue de l'étoile (figure 1).
Avec 4000 planètes découvertes en 20 ans dont plus de la moitié dans les trois dernières années, l'étude des planètes extrasolaires est devenue un thème majeur de l'astronomie moderne. La découverte de planètes différentes de celles observées dans notre système solaire interroge les modèles de formation et d'évolution des systèmes planétaires. Pour bien comprendre la nature de ces nouveaux objets, les données actuelles sont trop limitées et des grands projets notamment spatiaux seront nécessaires dans les années qui viennent.
Dès 1995, l'utilisation de spectromètres très stables avec des résolutions spectrales $>50000$ a permis de mesurer le décalage doppler provoqué par le mouvement d'une étoile autour du centre de masse du système étoile-planète. Cette technique mesurant les vitesses radiales des étoiles a permis la découverte de plus de 750 planètes. Encore plus productive, la méthode des transits a permis la détection de 3000 planètes mais avec des courtes périodes orbitales car la probabilité d'alignement Terre-Planète-étoile diminue rapidement quand la distance étoile-planète augmente.

Les premières détections indirectes ont permis la découverte de Jupiter chauds, des planètes de la taille de Jupiter orbitant très proches de leur étoile (figure 2) mais, statistiquement, leur présence autour d'une étoile de type solaire est plutôt rare $(0,5 \%$ à $1 \%)$. En revanche, la technique des transits a surpris par le grand nombre de petites planètes détectées avec des rayons de l'ordre de deux à trois fois celui de la Terre (figure 3).

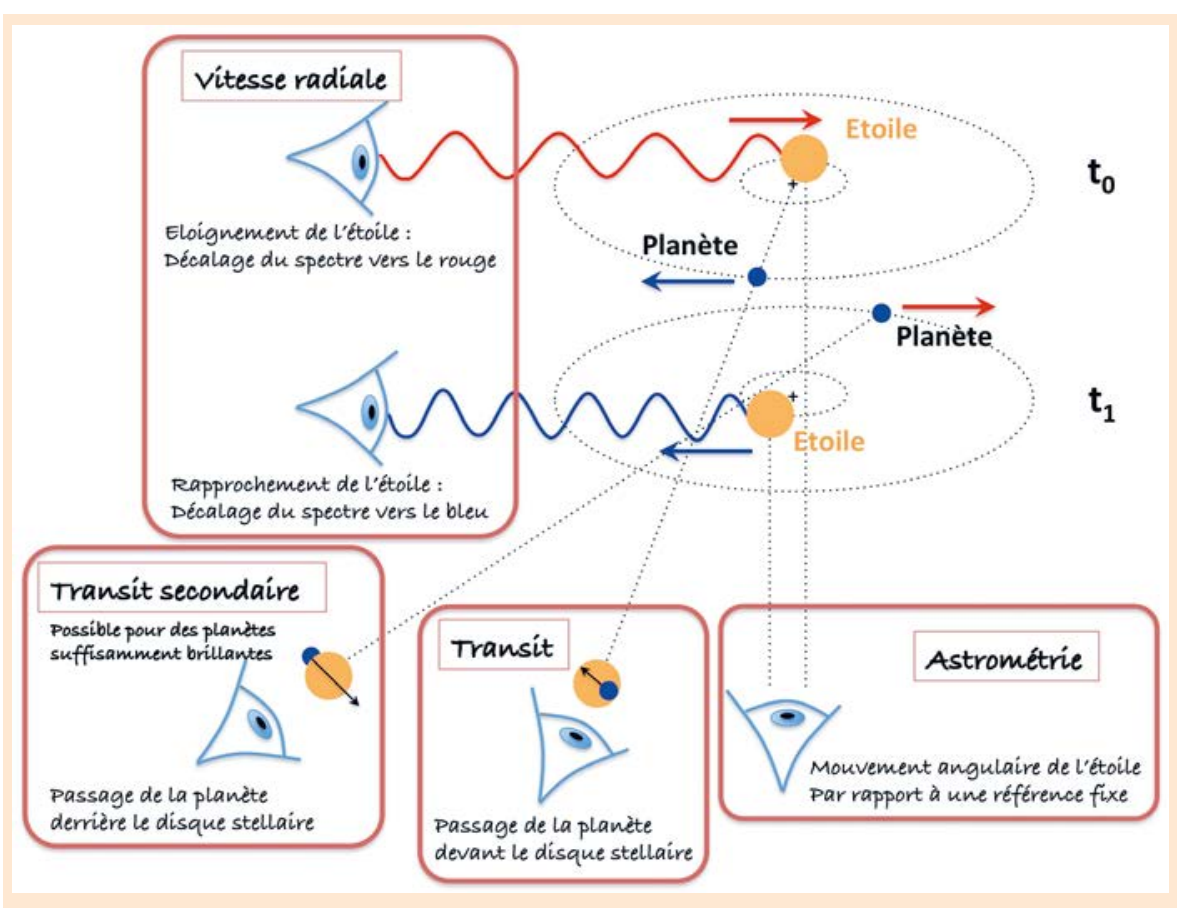

Figure 1. Description des principales méthodes de détection indirecte des planètes extrasolaires. 


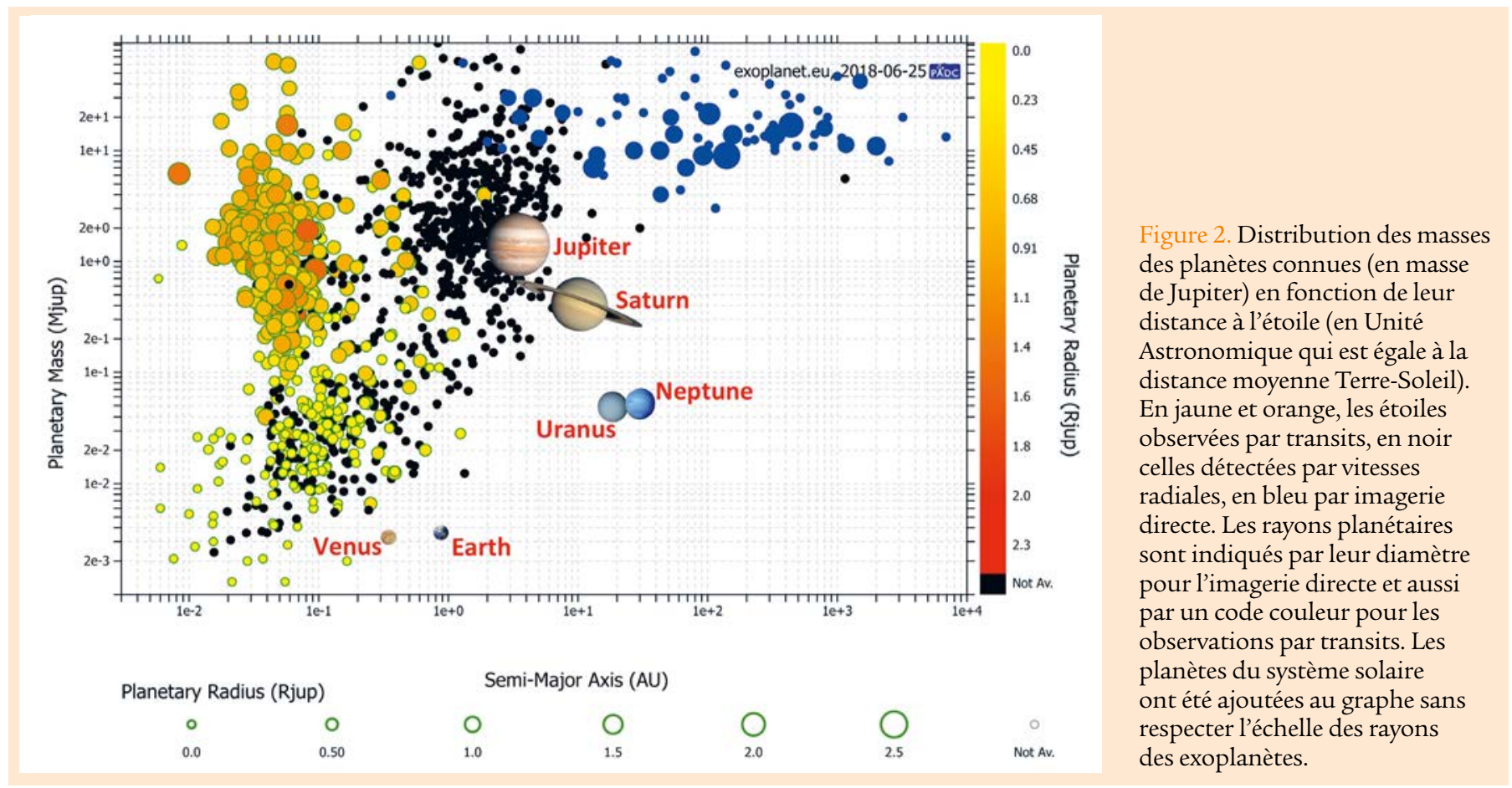

Les planètes détectées sur des orbites lointaines l'ont été par l'observation directe, c'est-à-dire en enregistrant une image de la planète séparée de son étoile sur un détecteur. A priori la solution la plus évidente, c'est la plus complexe à mettre en œuvre du fait de l'énorme rapport entre le signal reçu de l'étoile et celui provenant de la planète. Pour une planète de type Terre autour d'une étoile de type solaire, pour chaque photon reçu de la planète dans le visible, on reçoit dans le même temps $10^{10}$ photons de l'étoile. Le rapport peut être plus faible dans l'infrarouge : de l'ordre de $10^{7}$ à $10 \mu \mathrm{m}$ pour une Terre, ou de l'ordre de $10^{4}-10^{6}$ à $2 \mu \mathrm{m}$ pour des planètes géantes jeunes qui émettent encore une partie de l'énergie de leur formation. Ce sont quelques dizaines de ces géantes jeunes et éloignées de leur étoile qui ont été observées de manière directe par des instruments optimisés pour enregistrer des images avec des très grands contrastes.

\section{Vers une classification des planètes}

Aujourd'hui, notre connaissance sur toutes ces exoplanètes est limitée aux données orbitales et à des paramètres physiques de base (la masse, le diamètre et la densité moyenne). L'un des principaux enjeux dans les 10 ans qui viennent est d'obtenir des informations spectrales sur un échantillon représentatif des planètes observées afin de les classer en fonction de leur température d'équilibre, de leur masse et des composants de leur atmosphère (comme dans la figure 4). Un autre enjeu, lié au premier, est de mieux comprendre l'évolution de ces différentes planètes depuis leur formation jusqu'à leur mort. Un troisième point souvent mis en avant concerne les conditions d'apparition de la vie qui ne peut être vraiment résolu qu'en s'assurant de bien comprendre les deux points précédents.
Les seules techniques capables d'étudier les conditions (température, pression) et les composants de l'atmosphère sont, d'une part l'imagerie directe qui permet d'enregistrer le spectre de la lumière réfléchie par la planète dans le visible et de la lumière émise dans l'infrarouge, et d'autre part la spectroscopie des transits qui mesure la transmission de l'atmosphère de la planète au moment des transits, voire lorsque la planète passe derrière le disque stellaire (transits secondaires, figure 1).

\section{Des instruments spatiaux pour caractériser les exoplanètes}

Des grands projets d'observation sont en cours et ont été lancés pour répondre à ces questions. Pour les télescopes au sol, des instruments permettent déjà la découverte et la caractérisation des planètes comme l'instrument d'imagerie directe SPHERE ou l'instrument de mesure des vitesses radiales HARPS qui sont installés à l'Observatoire Européen Austral. D'autres instruments sont

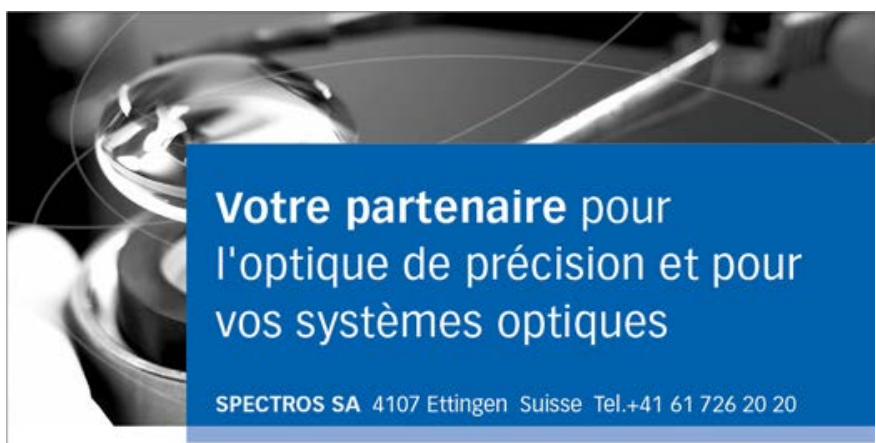

www.spectros.ch 


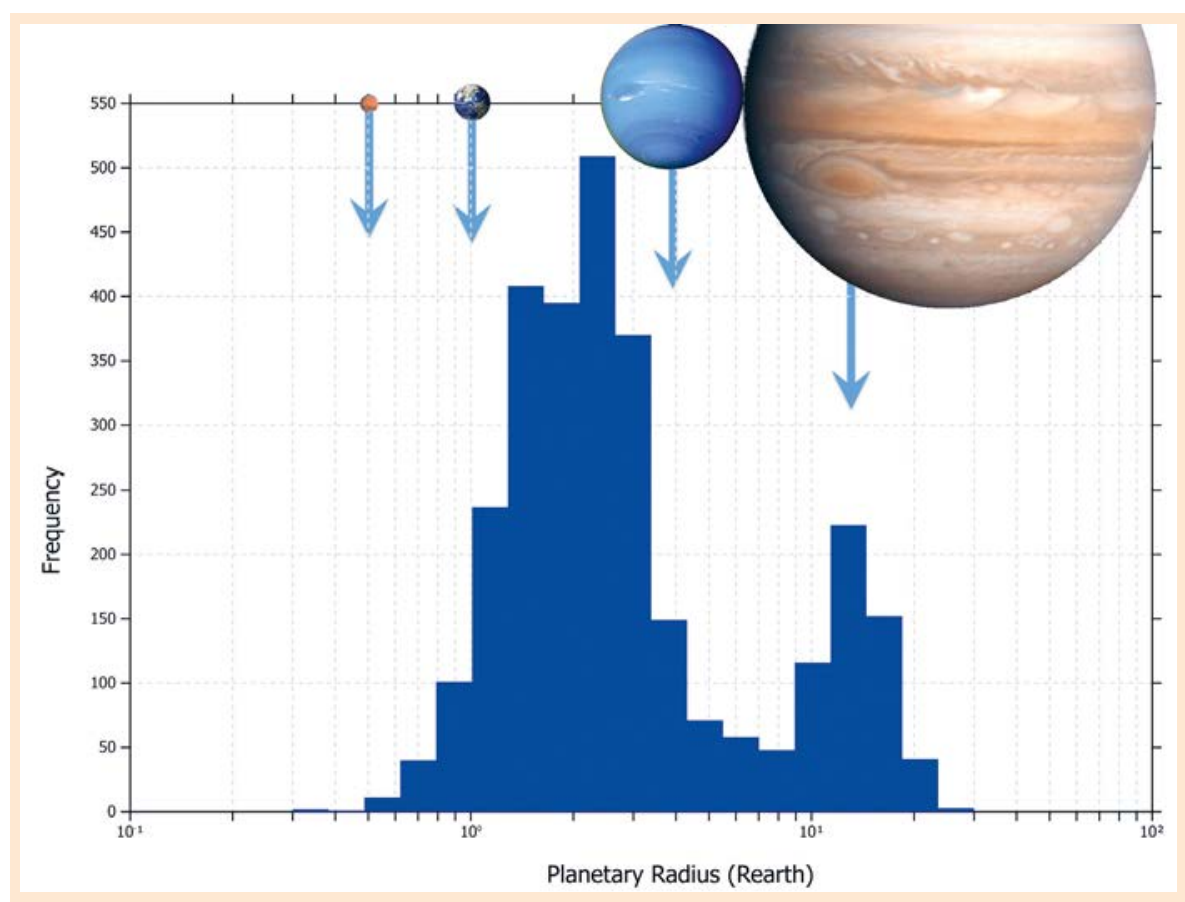

Figure 3. Nombre de planètes détectées par la méthode des transits en fonction du rayon planétaire mesuré. On voit un large pic entre 1 et 4 RTerre. Déduite de ces mesures, la probabilité de présence d'une planète de la taille d'une Super-Terre ou d'une Mini-Neptune autour d'étoiles peu massives est très grande (>80\%, [2]).

actuellement utilisés ou prévus sur les télescopes basés au sol mais une majorité des nouvelles planètes qui seront découvertes et caractérisées dans les 10 prochaines années le seront probablement par des missions spatiales.

Depuis l'espace, la détection des transits planétaires a été la technique d'observation la plus utilisée et la plus prolifique jusqu'ici avec une lignée de missions spatiales telles que CoRoT (CNES 2006-2014), Kepler (NASA, 2009-2018), TESS (NASA, 2018-) et CHEOPS (ESA, 2019). La mission astrométrique GAIA, lancée en 2013 et qui cartographie plus d'un milliard d'étoiles de notre galaxie, devrait découvrir plusieurs milliers de nouvelles planètes d'ici 2022 en déterminant leur masse avec précision. Parmi les futures missions de l'ESA, deux sont dédiées à l'étude statistique des propriétés des exoplanètes : PLATO et ARIEL.

PLATO (PLAnetary Transits and Oscillations of Stars), prévu pour un lancement en 2026, a pour objectif la recherche d'exoplanètes de toutes tailles par la méthode des transits. Sa caractéristique principale est l'utilisation de 24 caméras identiques dont les champs de vue se superposent partiellement, créant ainsi un champ de vue global du satellite supérieur à $2200 \mathrm{deg}^{2}$. Chaque caméra est entièrement dioptrique, avec une pupille de $120 \mathrm{~mm}$ et une distance focale de 200 $\mathrm{mm}$. Le plan focal de chaque caméra est constitué de 4 CCD de $4510 \times 4510$ pixels de $18 \mu \mathrm{m}$, soit une surface sensible du satellite supérieure à $0,6 \mathrm{~m}^{2}$ (2 $10^{9}$ pixels). Le projet s'approche d'une fabrication à la chaine avec la fourniture d'une caméra intégrée et alignée toutes les 2 semaines et une parallélisation des tests cryogéniques de chacune des caméras sur 3 sites en Europe. Le satellite est sous maitrise d'œuvre de la société OHB System AG avec des partenariats de Thales Alenia Space et RUAG Space. Scientifiquement, le couplage des mesures de transits avec des mesures de vitesses radiales sur les télescopes au sol permettra de fournir un catalogue de planètes avec des orbites assez longues ( 1 an) autour d'étoiles brillantes et proches qui pourront être utilisées comme cibles par les instruments de détection directe. PLATO aidera aussi à mieux comprendre les scénarios d'évolution des systèmes planétaires puisqu'il estimera simultanément l'âge du système planétaire par l'analyse sismique des étoiles hôtes comme l'avait fait CoRoT.

ARIEL est une mission de l'ESA dédiée à la spectroscopie des transits dont le lancement est prévu pour 2028. ARIEL comprendra un télescope de $1 \mathrm{~m}$ équipé $\mathrm{d}$ 'un spectromètre très stable offrant une couverture spectrale simultanée entre $0,5 \mu \mathrm{m}$ et $7,8 \mu \mathrm{m}$. L'objectif d'ARIEL est d'enregistrer les spectres d'un millier de planètes en transit afin de définir des tendances sur la composition chimique de leur atmosphère qui ne peuvent être révélées que de manière statistique.

Le télescope spatial James Webb (JWST) est le prochain observatoire astronomique multi-instruments avec des objectifs scientifiques très vastes allant de l'étude des premiers objets de l'Univers jusqu'aux origines des étoiles et des systèmes planétaires. Fruit de la coopération NASA-ESA, le JWST devrait remplacer dès 2021 le télescope spatial Hubble après 30 ans de bons et loyaux services. Son lancement a déjà été retardé plusieurs fois mais l'envoi d'un monstre de technologies de plus de 6 tonnes à plus d'1,5 million de kilomètres demande quelques précautions. Arrivé à sa destination située à plus de 4 fois la distance Terre-Lune, il n'y aura pas de possibilité de réparation par des astronautes comme sur Hubble. Pour rentrer dans la coiffe de la fusée Ariane 5, son miroir primaire de $6,5 \mathrm{~m}$ de diamètre (contre $2,4 \mathrm{~m}$ pour Hubble) va se mettre en quatre pour les astronomes. Le miroir est segmenté en 18 hexagones et replié en trois parties au moment du lancement et le reste du télescope est entièrement déployable et devra être cophasé avec une précision de l'ordre de 100 nanomètres. Le JWST observera dans les longueurs d'onde infrarouge (entre $0,6 \mu \mathrm{m}$ et $28 \mu \mathrm{m}$ ) qui sont optimales pour étudier les premiers objets de l'Univers mais également pour les exoplanètes qui présentent des rapports d'intensité moins extrêmes que dans le visible. Alors qu'ARIEL 
devrait permettre une classification méthodique des planètes avec des périodes relativement courtes (< 100 jours), le JWST, grâce à son large diamètre, devrait permettre d'obtenir les premiers spectres de quelques petites planètes très proches de leur étoile. Pour les planètes plus lointaines, le JWST observera sensiblement les mêmes planètes géantes jeunes actuellement étudiées avec les télescopes basés au sol mais pour des longueurs d'onde peu accessibles à travers l'atmosphère. Pour observer des planètes semblables à celles du système solaire, il faut des systèmes optiques beaucoup plus complexes qui vont supprimer la lumière diffractée de l'étoile d'un facteur $10^{9}$ $10^{11}$ à une séparation angulaire de quelques éléments de résolution (2-4 $\lambda / D)$. Ces techniques d'imagerie à haut contraste (voir l'article « Détection directe et caractérisation des planètes extrasolaires » dans Photoniques $\mathrm{n}^{\circ} 58$ ) permettent également l'enregistrement de spectre de planètes géantes et de planètes telluriques autour d'étoiles de type solaire. Le premier instrument qui devrait approcher cet objectif est la mission WFIRST.

WFIRST (Wide Field Infrared Survey Telescope) est une mission NASA avec quelques participations extérieures dont celle du CNES qui vise principalement à étudier la nature de l'énergie sombre et à recenser les exoplanètes de la galaxie par la méthode des microlentilles gravitationnelles. Le télescope de 2,4 m de diamètre non segmenté de WFIRST intègre également un instrument d'imagerie directe d'exoplanètes très ambitieux. La complexité de cet instrument et ses risques inhérents ont amené la NASA à définir cet instrument comme un démonstrateur de technologie avancée, c'est-à-dire que l'instrument intégrera des technologies novatrices généralement jamais testées dans l'espace, comme par exemple, deux miroirs déformables à grand nombre d'actionneurs $(48 \times 48)$, un module autonome d'analyse et de correction du front d'onde à des niveaux de précision d'une dizaine de picomètres, des coronographes à masques de phase complexes et une caméra hyperspectrale à faible bruit

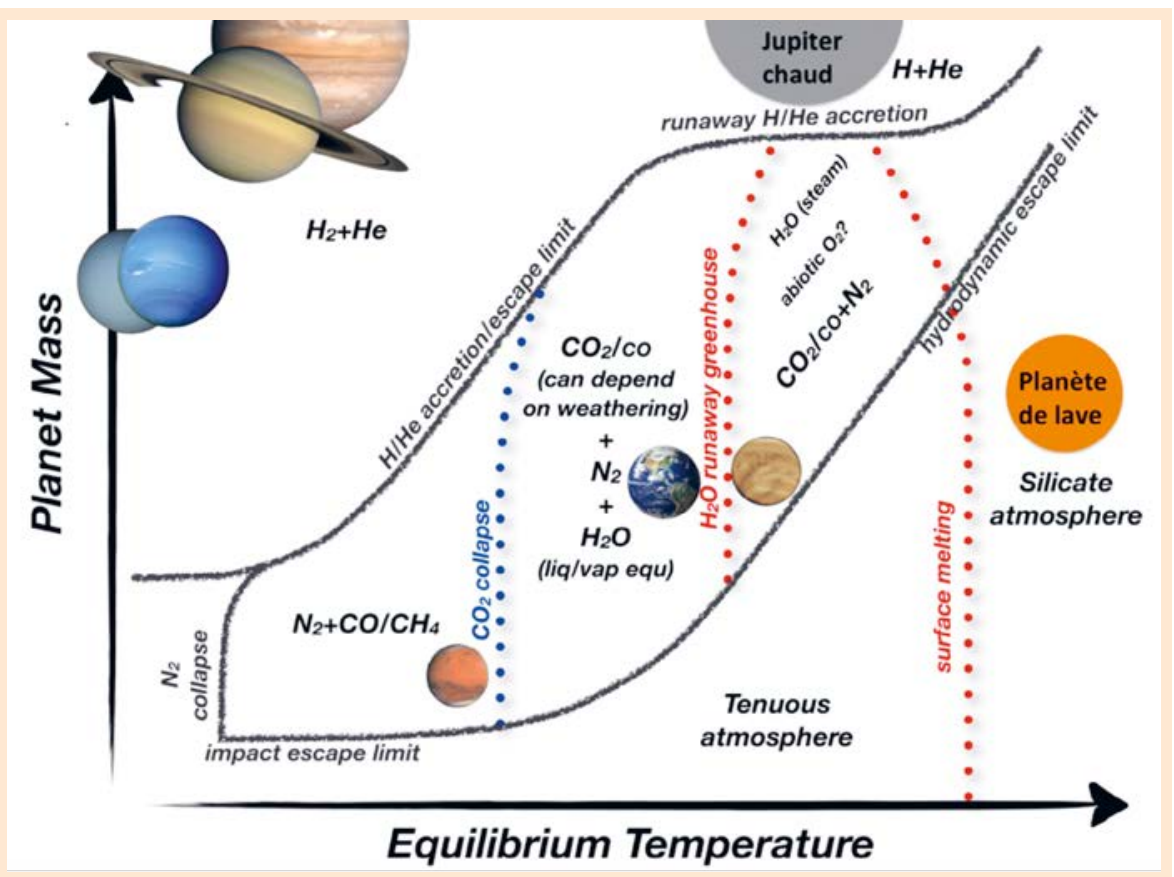

Figure 4. Diagramme des différentes classes d'atmosphères prédit par Leconte et al. (2014) [3]. Seules les espèces chimiques dominantes sont indiquées. Chaque ligne représente une transition d'un régime à un autre. La position et l'allure de ces «transitions » nécessitent des étalonnages à partir des observations. Les planètes du système solaire sont indiquées sur le diagramme ainsi que la position de quelques planètes plus exotiques.

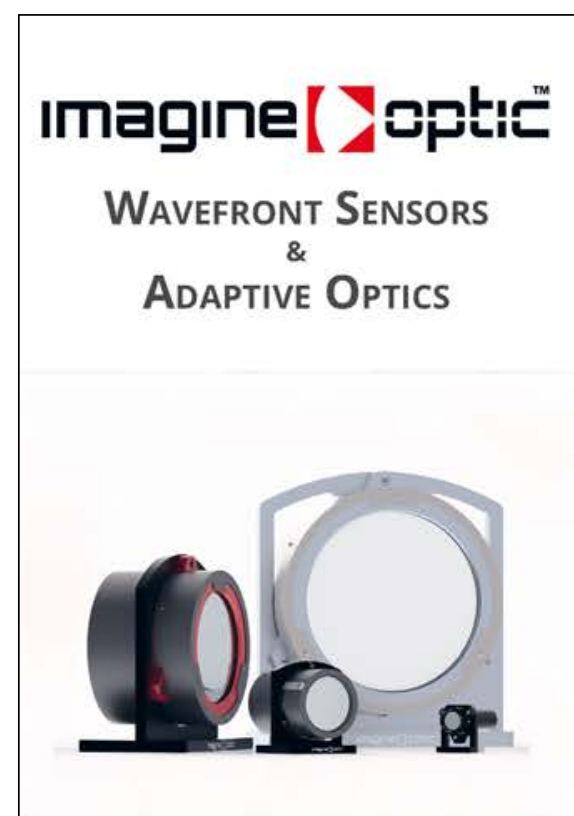

HIGH-POWERED LASER

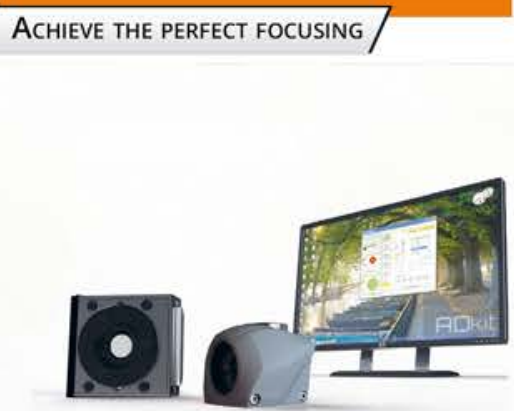

BIO IMAGING

CORRECT ABERRATIONS - IMPROVE IMAGING

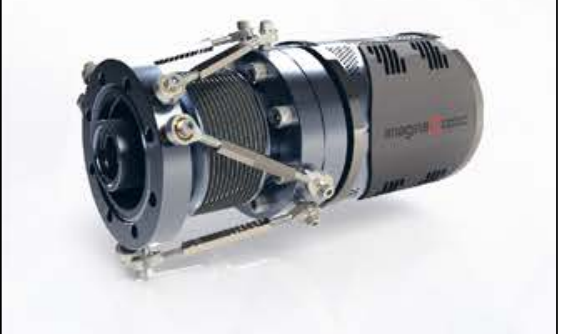

EUVIX-RAYS SENSING

ACtive WAVEFRONT CONTROL /

18 rue Charles de Gaule 91400 Orsay, France +33(0) 164861560 contact@imagine-optic.com

WWW.IMAGINE-OPTIC.COM 


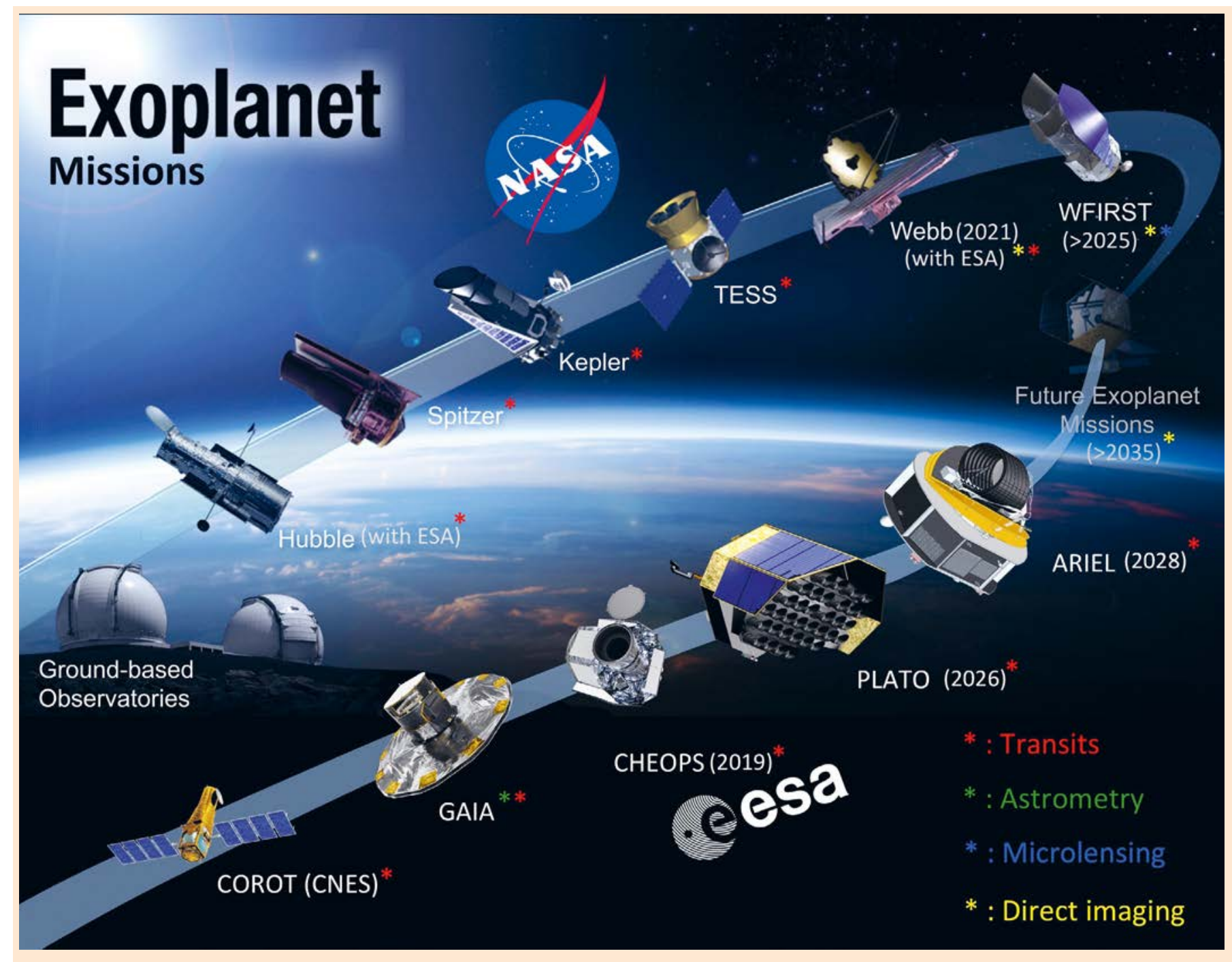

Figure 5. Principales missions spatiales dédiées ou partiellement dédiées aux exoplanètes. (Crédit : NASA/ESA)

de lecture. Ces technologies, à la pointe des développements actuels en laboratoire, seront intégrées sur le télescope qui est prévu pour un lancement après 2025. WFIRST devrait éteindre la lumière de l'étoile d'un facteur $10^{9}$ sur quelques bandes spectrales entre $550 \mathrm{~nm}$ et $1000 \mathrm{~nm}$. Ces performances déjà complexes à atteindre en laboratoire seront suffisantes pour obtenir la première caractérisation d'une atmosphère de géantes gazeuses semblables à Jupiter autour d'une étoile de type solaire et pourraient permettre l'étude de quelques planètes peu massives (mini-Neptune voire Super-Terre).

En revanche, l'étude des exoTerres devra sûrement attendre la génération de télescopes qui suivra le JWST. Quatre études de concept pour ce futur observatoire polyvalent ont été lancées en 2016 par la NASA avec deux télescopes particulièrement dédiés à l'observation d'exoplanètes telluriques. L'une des missions proposées est un télescope de $4 \mathrm{~m}$ de diamètre non-segmenté et hors-axe qui intègre un coronographe au foyer du satellite pour détecter les planètes telluriques dont l'étude spectrale se fait en couplant le télescope à un occulteur externe de $50 \mathrm{~m}$ de diamètre et lancé à $100000 \mathrm{~km}$ du télescope principal (mission Habex). L'autre étude propose des solutions basées sur des télescopes segmentés de large diamètre ( $8 \mathrm{~m}$ à $15 \mathrm{~m}$, mission LUVOIR). L'un de ces instruments qui devrait voir le jour d'ici 20 ans représentera l'aboutissement de développements en laboratoire et sur des instruments aussi bien spatiaux que basés au sol et devrait permettre d'aborder la question existentielle liée aux exoplanètes, celle des conditions d'apparition de la vie.

\section{POUR EN SAVOIR PLUS}

[1] Handbook of exoplanets, Deeg, Hans J., Belmonte, Juan Antonio (Eds.)

[2] N.M. Batalha, Exploring exoplanet populations with NASA's Kepler Mission, Proceedings of the National Academy of Sciences, vol. 111, pp. 12647-12654, 2014

[3] J. Leconte, F. Forget, H. Lammer, On the (anticipated) diversity of terrestrial planet atmospheres, Exp. Astronomy, vol. 40, pp. 449-467, 2015 